STRUCTURAL BIOLOGY COMMUNICATIONS

ISSN 2053-230X

\section{Structural insights into the catalytic reaction trigger and inhibition of D-3-hydroxybutyrate dehydrogenase}

\author{
Hiroki Kanazawa, ${ }^{a, b}$ Md. Mominul Hoque, ${ }^{b, c}$ Masaru Tsunoda, ${ }^{b}$ Kaoru Suzuki,

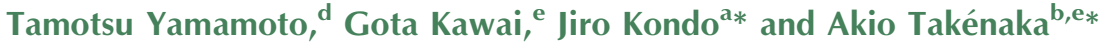

Received 2 February 2016

Accepted 11 May 2016

Edited by C. S. Bond, University of Western Australia, Crawley, Australia

Keywords: X-ray crystal structure; D-3-hydroxybutyrate dehydrogenase; substrate; inhibitor; catalytic reaction trigger; ketone bodies; acetyl-CoA.

PDB references: D-3-hydroxybutyrate dehydrogenase, complex with $\mathrm{NAD}^{+}$and D-3-hydroxybutyrate, 5b4t; complex with $\mathrm{NAD}^{+}$ and malonate, $5 \mathrm{~b} 4 \mathrm{u}$; complex with $\mathrm{NAD}^{+}$and methylmalonate, $5 \mathrm{~b} 4 \mathrm{v}$

Supporting information: this article has supporting information at journals.iucr.org/f

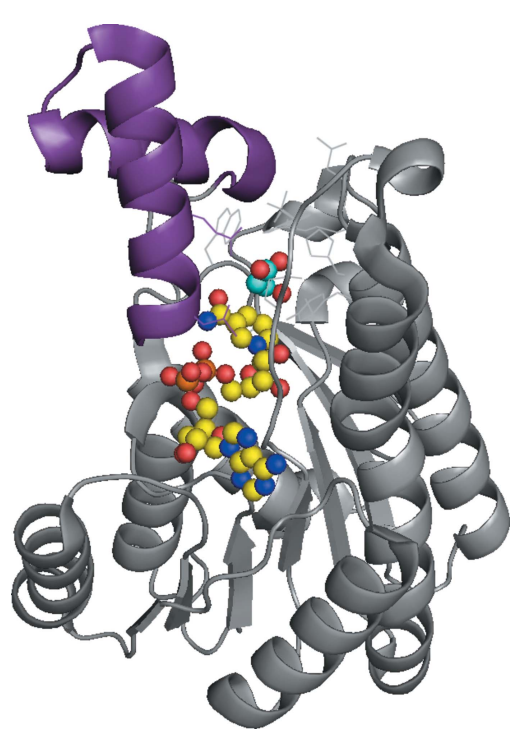

(C) 2016 International Union of Crystallography
aDepartment of Materials and Life Sciences, Sophia University, Kioi-cho, Chiyoda-ku, Tokyo 102-8554, Japan, ${ }^{\mathbf{b}}$ Faculty of Pharmacy, Iwaki Meisei University, Chuodai-iino, Iwaki 970-8551, Japan, ${ }^{\mathbf{c}}$ Department of Biochemistry and Molecular Biology, University of Rajshahi, Rajshahi 6205, Bangladesh, dAsahi Kasei Pharma Corporation, Tagata-gun, Shizuoka 410-2323, Japan, and ${ }^{\mathbf{e}}$ Research Institute, Chiba Institute of Technology, Tsudanuma, Narashino, Chiba 275-0016, Japan. *Correspondence e-mail: j.kondo@sophia.ac.jp, atakenak@sakura.email.ne.jp

D-3-Hydroxybutyrate dehydrogenase catalyzes the reversible conversion of acetoacetate and D-3-hydroxybutyrate. These ketone bodies are both energystorage forms of acetyl-CoA. In order to clarify the structural mechanisms of the catalytic reaction with the cognate substrate D-3-hydroxybutyrate and of the inhibition of the reaction by inhibitors, the enzyme from Alcaligenes faecalis has been analyzed by X-ray crystallography in liganded states with the substrate and with two types of inhibitor: malonate and methylmalonate. In each subunit of the tetrameric enzyme, the substrate is trapped on the nicotinamide plane of the bound $\mathrm{NAD}^{+}$. An OMIT map definitively shows that the bound ligand is D-3hydroxybutyrate and not acetoacetate. The two carboxylate $\mathrm{O}$ atoms form four hydrogen bonds to four conserved amino-acid residues. The methyl group is accommodated in the nearby hydrophobic pocket so that the formation of a hydrogen bond from the $\mathrm{OH}$ group of the substrate to the hydroxy group of Tyr155 at the active centre is facilitated. In this geometry, the $\mathrm{H}$ atom attached to the $\mathrm{C}^{3}$ atom of the substrate in the $s p^{3}$ configuration is positioned at a distance of $3.1 \AA$ from the nicotinamide $\mathrm{C}^{4}$ atom in the direction normal to the plane. In addition, the donor-acceptor relationship of the hydrogen bonds suggests that the Tyr $155 \mathrm{OH}$ group is allowed to ionize by the two donations from the Ser142 $\mathrm{OH}$ group and the ribose $\mathrm{OH}$ group. A comparison of the protein structures with and without ligands indicates that the Gln196 residue of the small movable domain participates in the formation of additional hydrogen bonds. It is likely that this situation can facilitate $\mathrm{H}$-atom movements as the trigger of the catalytic reaction. In the complexes with inhibitors, however, their principal carboxylate groups interact with the enzyme in a similar way, while the interactions of other groups are changed. The crucial determinant for inhibition is that the inhibitors have no active $\mathrm{H}$ atom at $\mathrm{C}^{3}$. A second determinant is the Tyr155 OH group, which is perturbed by the inhibitors to donate its $\mathrm{H}$ atom for hydrogen-bond formation, losing its nucleophilicity.

\section{Introduction}

There are two known pathways for the synthesis of acetylCoA, through glycolysis from glucose and through $\beta$-fatty acid oxidation from fatty acids (Laffel, 1999; Dedkova \& Blatter, 2014), both of which supply the acetyl group to the TCA cycle, as shown in Fig. 1. Excess acetyl-CoA is converted to acetoacetate $\left(\mathrm{AA}^{\mathbf{1}}\right)$ through three enzymatic steps in the liver; AA

\footnotetext{
${ }^{1}$ Abbreviations: HBDH, D-3-hydroxybutyrate dehydrogenase; AA, acetoacetate; HB, D-3-hydroxybutyrate; MA, malonate; mMA, methylmalonate; $\mathrm{Nc}$, nicotineamide moiety of $\mathrm{NAD}^{+}$. Amino-acid residues are referred to using the three-letter codes in the text and the one-letter codes in the figures.
} 
is then further converted reversibly to D-3-hydroxybutyrate (HB) by D-3-hydroxybutyrate dehydrogenase (HBDH) with NADH as a coenzyme, while a small amount of AA spontaneously converts to acetone. Fig. 2 shows the atomic numbering of the ligands. The HB, AA and acetone molecules are collectively named ketone bodies and are soluble in water. Acetone is excreted in exhaled breath. The remaining HB and AA are transported via the bloodstream to organs such as heart, kidney and brain as an energy source, where they are converted to acetyl-CoA. In other words, ketone bodies supply stored energy to organs that consume a large amount of energy.

An excess of ketone bodies in the blood causes ketoacidosis, as induced, for example, by diabetes mellitus (Laffel, 1999; Shimomura et al., 2013). The HB level is normally higher than the AA level (Koch \& Feldbruegge, 1987; Shimomura et al., 2013), but when the HB level decreases the symptoms of ketoacidosis are alleviated. Therefore, some inhibitors of HBDH function have been developed as medical drugs, for example malonate (MA), methylmalonate (mMA) etc. (Tan et al., 1975; El Kebbaj et al., 1980; Nakashima et al., 2009), although mMA causes some toxic symptoms (Tan et al., 1975). HBDH itself is used as a probe to detect the HB level in blood (Williamson et al., 1962; Harano et al., 1983; Koch \& Feldbruegge, 1987; Uno et al., 1987; Kwan et al., 2006; Shimomura et al., 2013; Pires et al., 2003). Structural insights into HBDH are essential in order to understand the reaction mechanism described above.

HBDH is a member of the large SDR (short-chain dehydrogenases/reductases) superfamily (Kavanagh et al., 2008; Persson \& Kallberg, 2013), which is widespread and originates from a common ancestor structure not only in mammals but in every living organism, including microorganisms. To understand the functional variety of protein structures from a phylogenic point of view, extensive structural studies have been performed. Therefore, several X-ray structures of HBDH have already been reported (Hoque et al., 2008, 2009; Nakashima et al., 2009; Paithankar et al., 2007; Ito et al., 2006; Guo et al., 2006), but the detailed enzymatic reaction mechanism and the substrate specificity are not yet fully understood. Previously, we suggested the substrate-binding mode using the structures of Alcaligenes faecalis $\mathrm{HBDH}$ in complex with $\mathrm{NAD}^{+}$and acetate (Hoque et al., 2009), and our proposal was subsequently confirmed by the crystal structure

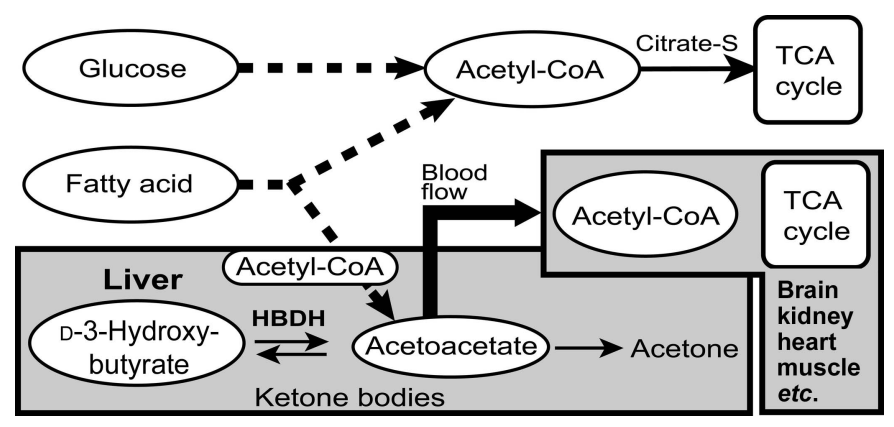

Figure 1

Flow of ketone bodies coupled to the acetyl-CoA supply system. of the ternary complex containing the cognate substrate $\mathrm{HB}$ (Hoque et al., 2008). However, owing to low resolution (3.0 $)$ it was difficult to distinguish clearly whether the bound molecule was HB or AA. In the present study, we have succeeded in obtaining another crystal form and have analyzed its structure at higher resolution $(1.19 \AA)$. In addition, to clarify the mechanism of inhibition of the catalytic reaction, crystal structures of $\mathrm{HBDH}$ complexed with $\mathrm{NAD}^{+}$ and either of two inhibitors, MA or mMA, have been determined.

\section{Materials and methods}

\subsection{Crystallization}

HBDH was expressed and purified using the method described in a previous report (Hoque et al., 2008), but the Q Sepharose FF column was renewed and the FF-type Phenyl Sepharose column was replaced by an HP-type column (GE Healthcare). The reaction activity was confirmed according to a published procedure (Shuster \& Doudoroff, 1962). Referring to the previous conditions, a sample of lyophilized $\mathrm{HBDH}$ was dissolved at a concentration of $20 \mathrm{mg} \mathrm{ml}^{-1}$ in $100 \mathrm{mM}$ Tris- $\mathrm{HCl}$ buffer $\mathrm{pH}$ 8.5. Crystallizations of three ternary complexes, $\mathrm{HBDH}-\mathrm{NAD}^{+}-\mathrm{HB}, \mathrm{HBDH}-\mathrm{NAD}^{+}-\mathrm{MA}$ and $\mathrm{HBDH}-\mathrm{NAD}^{+}-\mathrm{mMA}$, were carried out using the hangingdrop vapour-diffusion method by mixing equal volumes $(2 \mu \mathrm{l})$ of the enzyme solution and of reservoir solution containing $30-34 \%(w / v)$ PEG 4000, and then equilibrating the mixed solutions against $700 \mu \mathrm{l}$ reservoir solution in a 24-well plate (Stem Corporation, Japan) at $293 \mathrm{~K}$. Crystals of the HBcontaining complex (HB complex), the MA-containing complex (MA complex) and the mMA-containing complex (mMA complex) were easily obtained within a few days at $293 \mathrm{~K}$ when the protein solution consisted of $5 \mathrm{mM} \mathrm{NAD}{ }^{+}$and $200 \mathrm{~m} M$ sodium salt of $\mathrm{HB}$, MA or mMA. The actual $\mathrm{pH}$ values of the droplets were within the range 7.7-8.0.

\subsection{X-ray diffraction data}

Fresh crystals were transferred into cryoprotectant solution (a 1:1 mixture of the reservoir solution and $80 \%$ glycerol solution) for $30 \mathrm{~s}$ and mounted on a Cryo-Loop (Hampton Research, California, USA) for flash-cooling in a cold nitrogen-gas stream at $100 \mathrm{~K}$. X-ray diffraction from crystals of the three complexes was recorded at $100 \mathrm{~K}$ on a Quantum 315r detector (ADSC, California, USA) using synchrotron radiation $(\lambda=1.00 \AA)$ on BL-5A of the Photon Factory (PF),
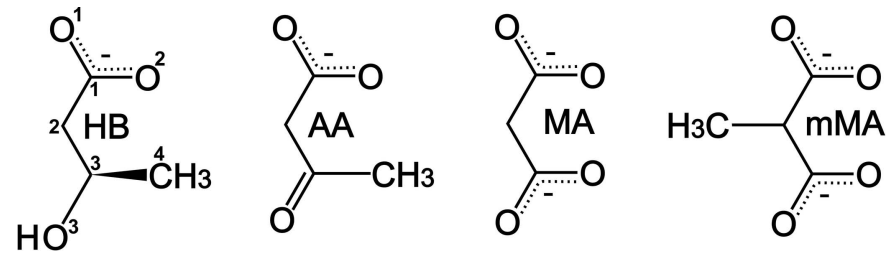

Figure 2

Chemical structures of HB, AA, MA and mMA. The numbers indicate the atomic numbering used in the text. 
Table 1

Crystal data, data-collection statistics and structure-refinement statistics.

Values in parentheses are for the highest resolution shell.

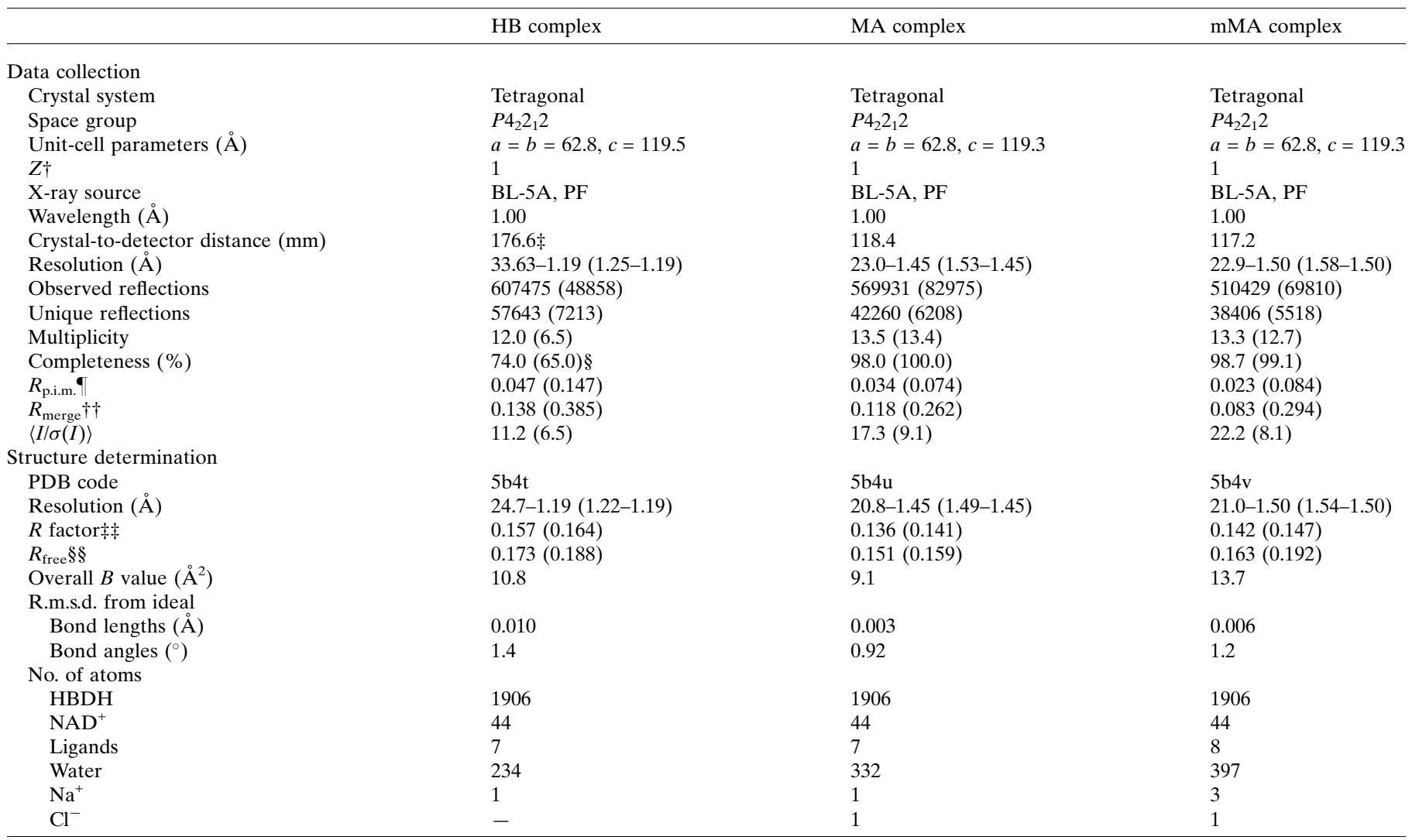

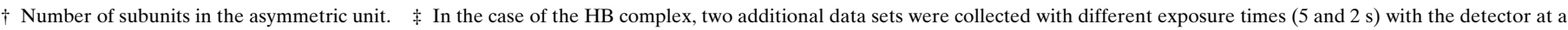

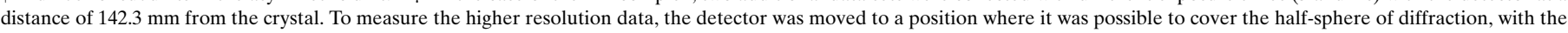

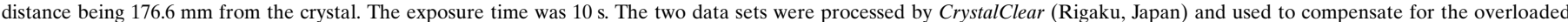

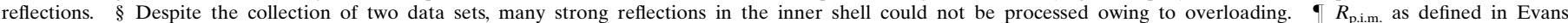

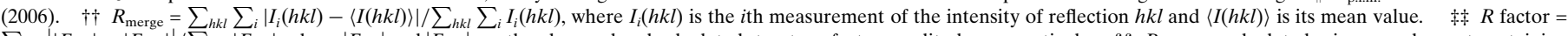

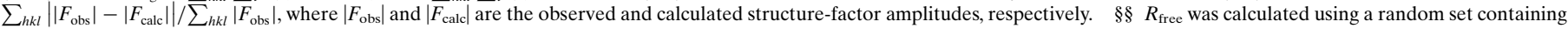
$5 \%$ of the observations that were not included throughout refinement (Brünger, 1992).

Tsukuba, Japan. Crystals diffracting to higher resolution were chosen for complete data collection. All diffraction patterns of these crystals were obtained with $1^{\circ}$ oscillation steps with $10 \mathrm{~s}$ exposure per each frame; a total of 180 frames were recorded by rotating the crystal samples. In the case of the HB complex, however, the 360 frames were taken to cover the half reciprocal sphere at the highest resolution. Bragg spots were indexed and their intensities were integrated using iMosflm (Battye et al., 2011). The collected intensity data were scaled between the frames and were then converted to amplitudes using SCALA (Evans, 2006) in CCP4 (Winn et al., 2011). In the case of the HB complex, some overloaded reflections were compensated for by merging with two additional data sets obtained using different exposure times at different distances from the crystals. Table 1 lists the diffraction-intensity processing statistics and the crystallographic data.

\subsection{Structure determination}

Approximate phase angles for the three data sets were derived by the molecular-replacement method with MOLREP
(Vagin \& Teplyakov, 2010) and AMoRe (Navaza, 1994) in CCP4 using a subunit structure of tetrameric HBDH (PDB entry $3 \mathrm{vdr}$; Hoque et al., 2009) as a reference structure. To find the remaining bound molecules, the atomic parameters were refined using REFMAC5 (Murshudov et al., 1997, 2011) and CNS (Brünger et al., 1998; Brünger, 2007). After the addition of $\mathrm{NAD}^{+}$and $\mathrm{HB}, \mathrm{MA}$ or mMA molecules, the atomic parameters of each HBDH complex were further refined together with additional solvent water molecules and ions. The statistics of structure refinement are summarized in Table 1. Coot (Emsley et al., 2010) from the CCP4 suite was used for the manipulation of electron-density maps. The final $F_{\mathrm{o}}-F_{\mathrm{c}}$ OMIT maps of $\mathrm{HB}$, the Nc moiety of $\mathrm{NAD}^{+}, \mathrm{MA}$ and mMA are shown in Fig. 3. These figures were generated by $D I N O$ (Philippsen, 2003) and Figs. 4-8 were generated by PyMOL (DeLano, 2002), with the exception of Fig. 7(a), which was drawn using Coot. A Ramachandran plot shows that the the $\varphi / \psi$ angles in favoured and allowed regions are 98.2 and $1.8 \%$, respectively, for the HB complex, 97.7 and $1.9 \%$, respectively, for the MA complex and 98.2 and $1.6 \%$, respectively, for the mMA complex. 


\section{Results and discussion}

\subsection{Overall structures}

When the present HB complex is compared with the previously published HB complex (Hoque et al., 2009), it is notable that the two crystal forms are quite different, even though they were obtained under similar conditions. The space group and unit-cell parameters of the previous structure are $P 4_{1} 2_{1} 2$ and $a=b=91.6, c=261.0 \AA$, while those of the present structure are $P 4_{2} 2{ }_{1} 2$ and $a=b=62.8, c=119.5 \AA$, respectively. In the present work, the crystal structures of the HB complex, MA complex and mMA complex have been determined at higher resolutions of $1.19,1.4$ and $1.5 \AA$, respectively. Therefore, as shown in Fig. 3, their $F_{\mathrm{o}}-F_{\mathrm{c}}$ OMIT maps are well resolved to identify the molecular structures; in particular, the HB complex shows electron density at the atomic level, in contrast to the previous $3.0 \AA$ resolution map.

Fig. 4(a) shows the overall tetrameric structure of $\mathrm{HBDH}$ composed of four subunits related by crystallographic 222 symmetry around $\left(\frac{1}{2}, \frac{1}{2}, 0\right.$ ) (for the side view see Supplementary Fig. S1). Each subunit is divided into two parts: the large domain (residues 1-190 and 220-260) and the small movable domain (residues 191-219). In the present complexes, all of the subunits adopt a closed conformation as the NAD ${ }^{+}$-bound state, as shown in Fig. 4(b). This feature is common to the three complexes, and the r.m.s.d.s on $\mathrm{C}^{\alpha}$ atoms on superimposition are $0.11 \AA$ between the HB complex and the MA complex and $0.15 \AA$ between the HB complex and the mMA complex, as shown in Fig. 4(c). Since the $\mathrm{p} K_{\mathrm{a}}$ values of $\mathrm{HB}$, AA, MA and mMA are 4.41, 4.02, 2.43 and 2.48, respectively, their carboxy groups must be dissociated in the crystalline state.

\subsection{Substrate binding and catalytic reaction}

The final OMIT map (Fig. 3a) definitively shows that the bound molecule is D-3-hydroxybutyrate (HB) and not aceto- acetate. Consistently, the OMIT map of the Nc moiety (Fig. $3 b$ ) is planar, indicating that the cofactor is $\mathrm{NAD}^{+}$and not NADH. HB is trapped on the nicotinamide plane of the $\mathrm{NAD}^{+}$bound in the central part of $\mathrm{HBDH}$, as shown in Figs. 4(b), 5(a) and 5(b). The two carboxylate $\mathrm{O}$ atoms of $\mathrm{HB}$ interact with $\mathrm{HBDH}$ through four hydrogen bonds. One $\mathrm{O}$ atom accepts the two carbamoyl NH groups of Gln94 and Gln196; Gln196 belongs to the small movable domain. Another $\mathrm{O}$ atom also forms two hydrogen bonds to Lys152 and His144. These hydrophilic interactions are the same as those found in the HBDH-NAD ${ }^{+}$-acetate complex (Hoque et al., 2008).

Near to the reaction centre, the hydroxy group attached to the $\mathrm{C}^{3}$ atom of $\mathrm{HB}$ is specifically recognized through two hydrogen bonds, one accepting the hydroxy group of Ser142 and the other donating its hydroxy group to Tyr155, as shown in Fig. 5(b). The remaining methyl group attached to the $\mathrm{C}^{3}$ atom of $\mathrm{HB}$ is accommodated in a hydrophobic pocket surrounded by the methyl group of Ala143 and the planar moieties of the Trp187, Trp257 and His144 residues. Thus, the space around the $\mathrm{C}^{3}$ binding site seems to be suitable for the $\mathrm{C}^{3}$ atom to adopt the $s p^{3}$ configuration. Based on this geometry, it is easy to estimate the position of the $\mathrm{H}$ atom attached to $\mathrm{C}^{3}$, as shown in Fig. 5(b) as an arrow towards the $\mathrm{C}^{4}$ atom of Nc. The $\mathrm{C}^{3}-\mathrm{H}$ bond vector is almost perpendicular to the Nc plane and points in the direction of the $\mathrm{C}^{4}$ atom of $\mathrm{Nc}$, so that the two atoms, $\mathrm{C}^{3}$ of $\mathrm{HB}$ and $\mathrm{C}^{4}$ of Nc, closely approach each other at a distance of $3.1 \AA$, suggesting a trigger of the catalytic reaction.

It can also be noted that the hydroxy $\mathrm{O}$ atom of Tyr155 forms another hydrogen bond to the hydroxy $\mathrm{O}^{2^{\prime}}$ atom attached to $\mathrm{C}^{2^{\prime}}$ of the $\mathrm{NAD}^{+}$ribose. Since the $\mathrm{O}^{2^{\prime}}$ atom accepts an $\mathrm{O} \cdots \mathrm{HN}$ hydrogen bond from the amino group of Lys159, this $\mathrm{O}^{2^{\prime}}$ atom could donate an $\mathrm{H}$ atom as a donor for hydrogen-bond formation. Therefore, the hydroxy $\mathrm{O}$ atom of Tyr155 must form two hydrogen bonds to bifurcated acceptors

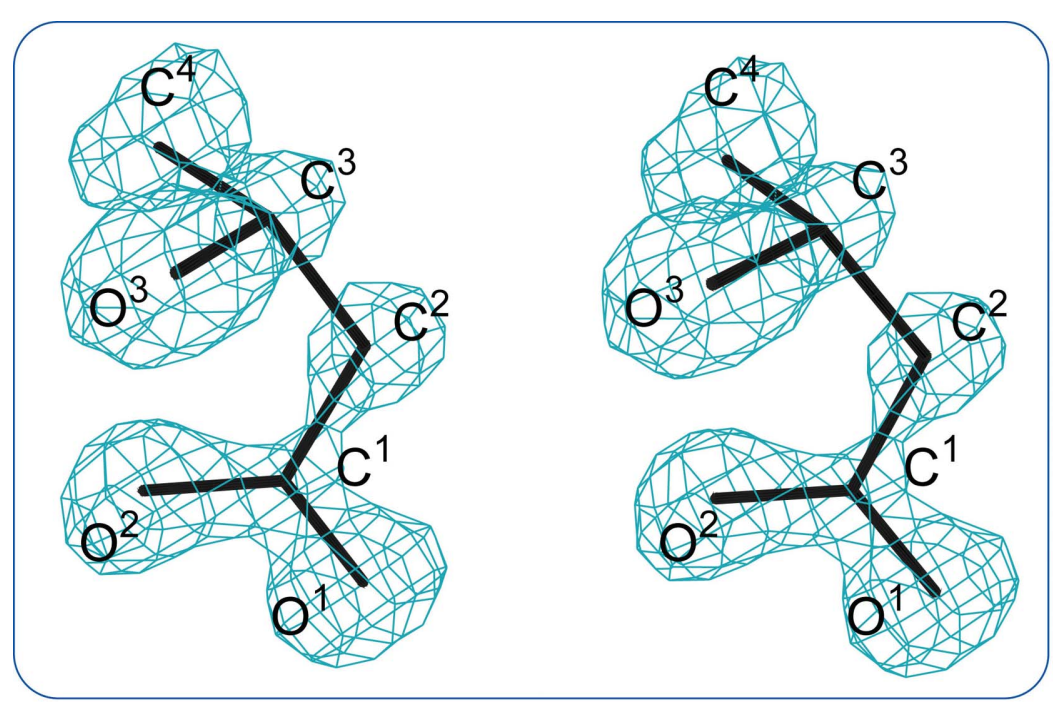

Figure 3

(a)
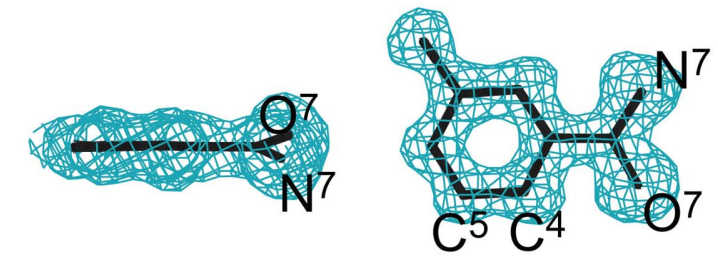

(b)

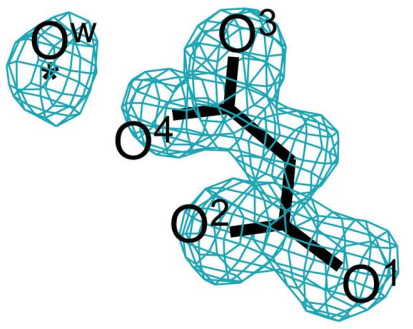

(c)

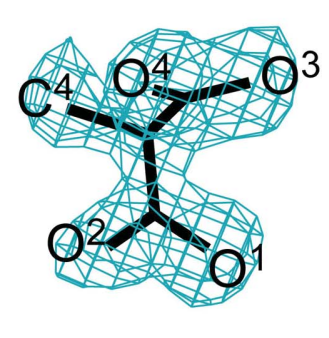

(d)

OMIT $F_{\mathrm{o}}-F_{\mathrm{c}}$ maps of HB (stereo pair) $(a), \mathrm{Nc}(b)$, MA $(c)$ and mMA $(d)$. Electron density is contoured at $2.5-3 \sigma$. The asterisk in $(c)$ indicates an isolated water $\mathrm{O}$ atom that was incorporated to fill the void space of the hydrophobic pocket upon MA binding. 
from the ribose $\mathrm{OH}$ group and from the $\mathrm{HB} \mathrm{OH}$ group, because the latter accepts the OH group of Ser142 (see Figs. $6 a$ and $6 c$ ). These hydrogen bonds suggest ionization of the Tyr 155 residue, releasing a proton at the crystallization $\mathrm{pH}$, as shown in Fig. 6. This subtle change might be related to the movement of $\mathrm{H}$ atoms during catalysis. The $\mathrm{H}$ atom of $\mathrm{C}^{3}$ could easily attack the $\pi$ orbital of the $\mathrm{C}^{4}$ atom of Nc, while the $\mathrm{H}$ atom of the hydroxy group of $\mathrm{HB}$ could move to the deprotonated $\mathrm{O}$ atom of Tyr155 following conversion from $\mathrm{HB}$ to AA. The reversed double $\mathrm{H}$-atom movements at $\mathrm{C}^{3}$ and Tyr155 are also applicable to the reverse reaction from AA to HB. Thus, the geometrical features of the substrate-binding site can accept only D-form 3-hydroxybutyrate with the $R$ configuration at the $\mathrm{C}^{3}$ atom. Even if the L-form (L-HB) is bound, the catalytic reaction does not proceed because the $\mathrm{H}$ atom of $\mathrm{C}^{3}$ is located in the opposite direction to $\mathrm{Nc}$, as

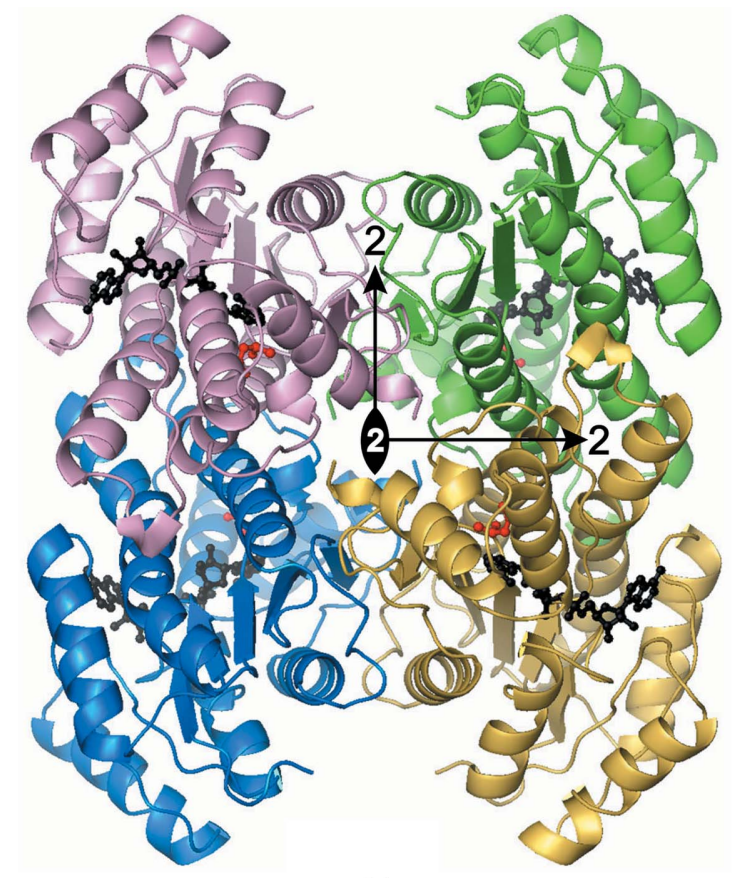

(a)

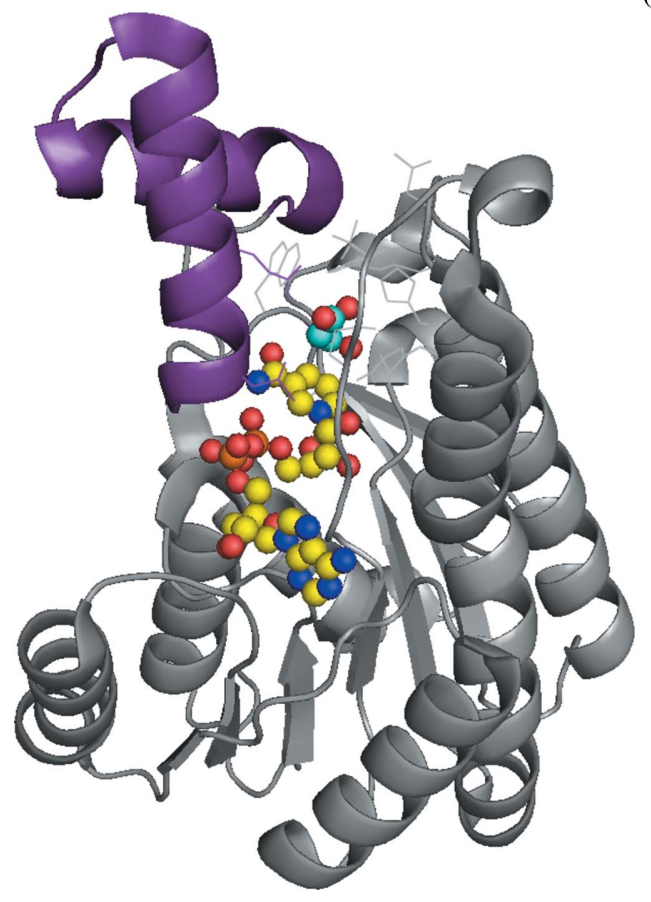

(b)

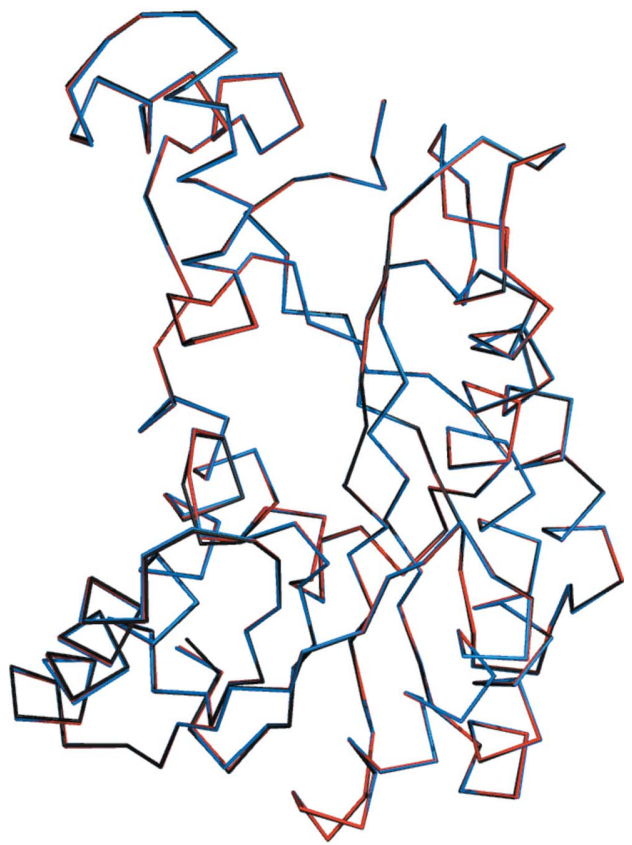

(c)

Figure 4

Overviews of the tetrameric HBDH structure with 222 symmetry viewed along $(1,-1,0)(a)$ and the subunit $(b)$. The small movable domain (violet) interacts with $\mathrm{NAD}^{+}$(yellow) and $\mathrm{HB}$ (cyan). Thin lines indicate the side chains surrounding HB. For conformational comparison $(c)$, the $\mathrm{C}^{\alpha}$ atoms of the MA complex (red) and the mMA complex (blue) are superimposed on those of the HB complex (black). Their r.m.s.d. values are 0.11 and $0.15 \AA$, respectively, suggesting no significant conformational changes upon complex formation with substrate/inhibitors. 
observed in a complex with the L-form (Nakashima et al., 2009). These residues shown in Figs. 5 and 6 are highly conserved between organisms as the essential amino acids for the catalytic reaction of the enzyme (Mountassif et al., 2010).

In the structural analysis of the previous $\mathrm{HB}$ complex ( $P 4_{1} 2_{1} 2$ form; Hoque et al., 2009), it was difficult to distinguish the configuration of the bound molecule between $\mathrm{HB}$ and AA owing to low resolution. In contrast, the present HB-complex crystal ( $P 4_{2} 2_{1} 2$ form) indicates that $\mathrm{HB}$ is not converted to AA in the crystalline state even near the optimal $\mathrm{pH}$ of the catalytic condition. In addition to the space groups, the packing densities also differ, as is apparent from the solvent contents: $51.2 \%\left(V_{\mathrm{M}}=2.52 \AA^{3} \mathrm{Da}^{-1}\right)$ for the $P 4_{1} 2{ }_{1} 2$ form and $43.3 \%$ $\left(V_{\mathrm{M}}=2.17 \AA^{3} \mathrm{Da}^{-1}\right.$ ) for the $P 4_{2} 2_{1} 2$ form. It is of further interest to speculate why the catalytic reaction does not occur in the present crystal. In the purification of the present $\mathrm{HBDH}$ sample, two chromatographic columns were renewed. As soon as fresh crystals appeared, they were quickly cooled for X-ray data collection. These treatments, especially the purity of the HBDH sample, may have caused the difference in the crystal form.

In a comparison of the two crystal structures, it has been found that the molecular interactions around the reaction centres are quite different. Fig. 7(a) shows that the movable domain composed of the helix-turn-helix is extruded into the solvent region without any interactions in the $P 4_{1} 2{ }_{1} 2$ form. In the $P 4_{2} 2_{1} 2$ form (Fig. $7 b$ ), however, each HBDH subunit of a tetramer is associated with the subunit of a neighbouring tetramer related by crystallographic twofold symmetry. In this association, the movable small domain interacts with a helix of the remaining large domain belonging to another tetramer through several direct hydrogen bonds (see Supplementary Fig. S2) and hydrophobic contacts. These interactions would affect the position of the small domain that carries Gln196, which is essential for substrate recognition. This situation is shown in Fig. 6(b), which shows 13 superposed HBDH subunits: five in complex with NAD and $\mathrm{HB}$, and the remaining eight in the apo state (PDB entries $2 \mathrm{yz} 7$ and $3 \mathrm{vdq}$ ). Although the small domain is highly variable in the apo state, this domain is moved to close the active site in the complex with the ligands, and the Gln196 side chain moves closer to the substrate to form a hydrogen bond (for details, see Fig. 6c). When the domain movement is affected by the molecular packing, its influence will further propagate to the interaction of $\mathrm{HB}$ and Nc. Here, it is interesting to speculate that the $\mathrm{C}^{3} \cdots \mathrm{C}^{4}$ distance will change depending on the movement of the small domain. In other words, the flexibility of the small domain might be coupled to the catalytic reactions in both

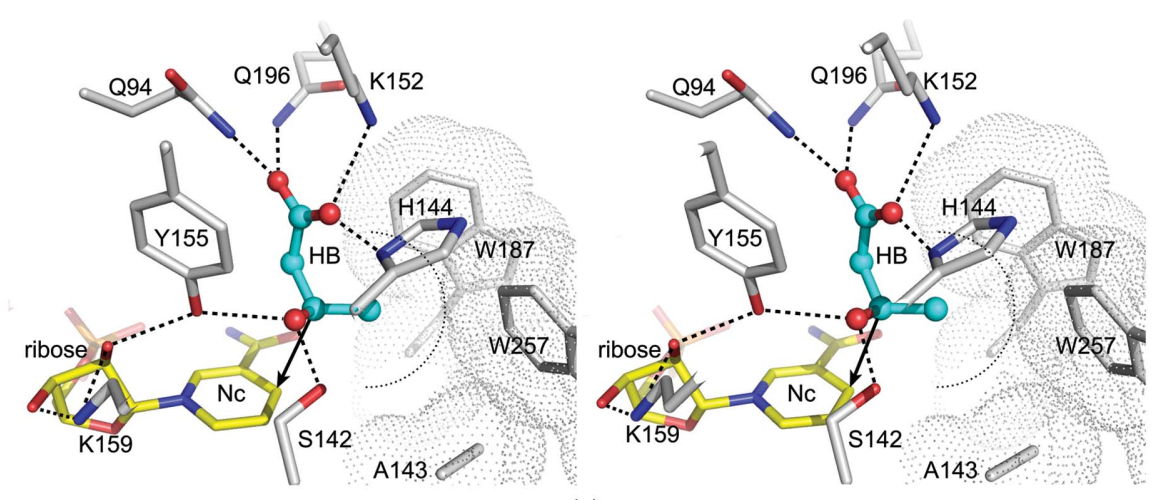

(a)

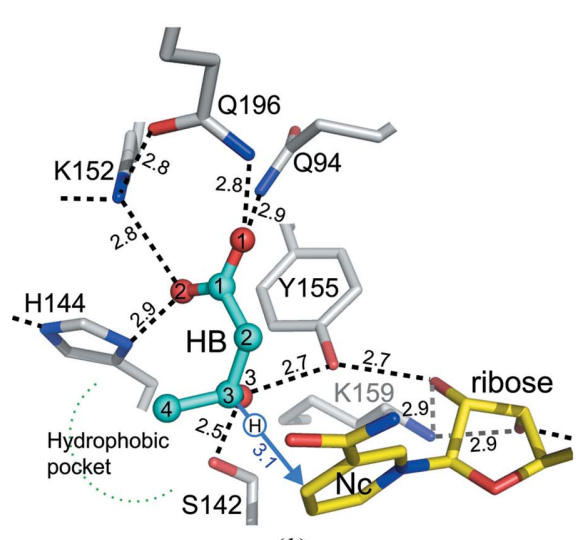

(b)

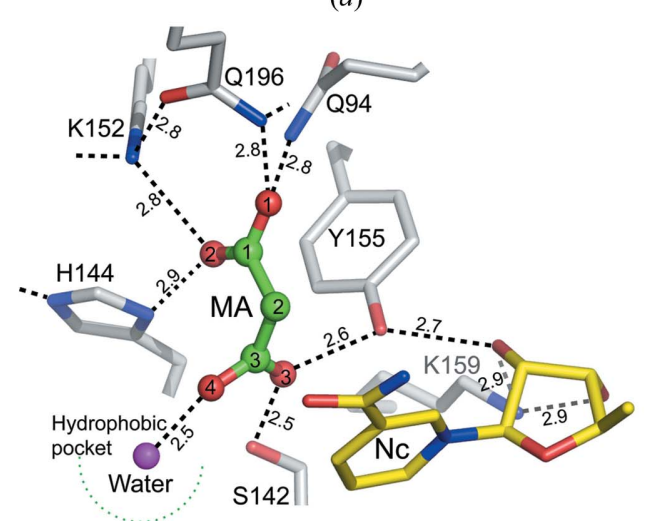

(c)

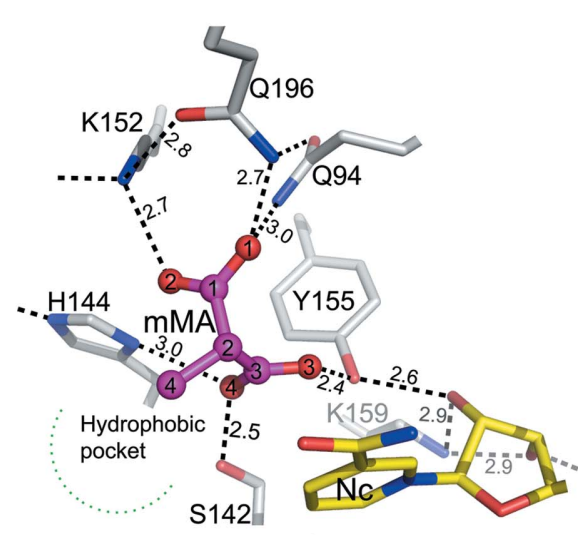

(d)

Figure 5

(a) A stereo-pair diagram of the HB-bound state of HBDH. $(b-d)$ Detailed interaction geometries of the substrate/inhibitor-binding sites found in the HB complex (b), MA complex $(c)$ and mMA complex $(d)$ projected from the back side of $(a)$. Broken lines indicate possible hydrogen bonds. Dotted half circles show the hydrophobic pocket surrounded by His144, Ala143, Trp187 and Trp257 that accepts the methyl group of HB in the HB complex, the carboxylate $\mathrm{O}$ atom of MA and a water molecule in the MA complex and a methyl group of mMA. One-letter codes are employed to specify the aminoacid residues. The numbers on the spheres indicate the atom numbering. An $\mathrm{H}$ atom in a blue circle, postulated to fill the tetrahedral configuration of the $\mathrm{C}^{3}$ atom, points towards the $\mathrm{C}^{4}$ atom of $\mathrm{Nc}$ in $(b)$, with a $\mathrm{C}^{3} \cdots \mathrm{C}^{4}$ distance of $3.1 \AA$. 
directions: from $\mathrm{HB}$ to $\mathrm{AA}$ and from $\mathrm{AA}$ to $\mathrm{HB}$. Some differences in the flexibility between the two forms can be estimated from the atomic r.m.s.d. values of their structures. When the four subunits of the $P 4_{1} 2_{1} 2$ form are superimposed on the present subunit, the values are $0.89 \AA$ between all atoms including the side chains and $0.53 \AA$ between the $\mathrm{C}^{\alpha}$ atoms. Indeed, the latter value is significantly larger than the corresponding values of 0.11 and $0.15 \AA$ when compared with the MA complex and mMA complex (see Fig. $4 c$ ) crystallized in the same $P_{2} 2_{1} 2$ form. Therefore, it could be concluded that the molecular interactions suppress the catalytic reaction in the $P 4_{2} 2_{1} 2$ packing with the help of the excess amount of substrate molecules $\left(200 \mathrm{~m} M\right.$ compared with $5 \mathrm{mM} \mathrm{NAD}^{+}$).

In order to confirm the configurational change of the $\mathrm{HB} \mathrm{C}^{3}$ atom, X-ray/neutron analyses could visualize the stepwise

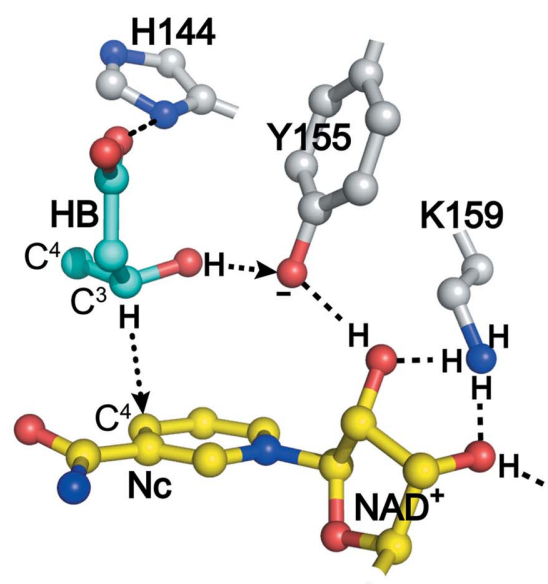

(a)

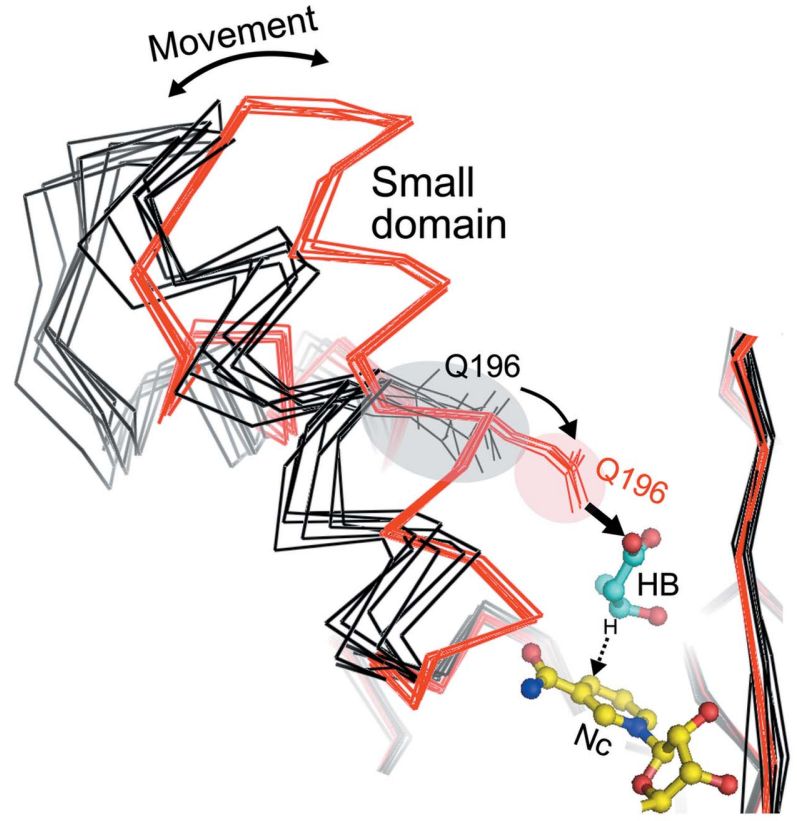

(b) structural changes during the catalytic reaction along with $\mathrm{pH}$ changes in the crystalline state. In addition, if the HB-complex crystal diffracts well beyond $1 \AA$ resolution, the $\mathrm{H}$-atom movement described above could be demonstrated by X-ray/ neutron analysis.

\subsection{Inhibitor binding and inhibition of the catalytic reaction}

As shown in Fig. 2, MA and HB differ from each other in the attachment of carboxy or 1-hydroxyethyl functional groups to the $\mathrm{C}^{2}$ atom of acetate. MA and AA are similar to each other in molecular shape, but the latter contains a large hydrophobic methyl group at $\mathrm{C}^{3}$. Since HB should adopt the $R$ configuration (D-form) at the $\mathrm{C}^{3}$ atom, the $\mathrm{L}$-form acts as an inhibitor (Nakashima et al., 2009). Furthermore, mMA and MA differ in the presence or absence of a methyl group at the $\mathrm{C}^{2}$ atom. Compared with the HB-complex crystals, there are no significant changes in the conformation of the $\mathrm{HBDH}$ folding and of the bound $\mathrm{NAD}^{+}$molecules between MAcomplex and mMA-complex crystals.

Fig. 8(a) shows the structures of the bound-state HB, MA and mMA molecules superimposed on the principal carboxylate groups for comparison. In the MA complex (Fig. 5c), MA is trapped in the central part of $\mathrm{HBDH}$, where the principal carboxylate group corresponding to that of HB is firmly

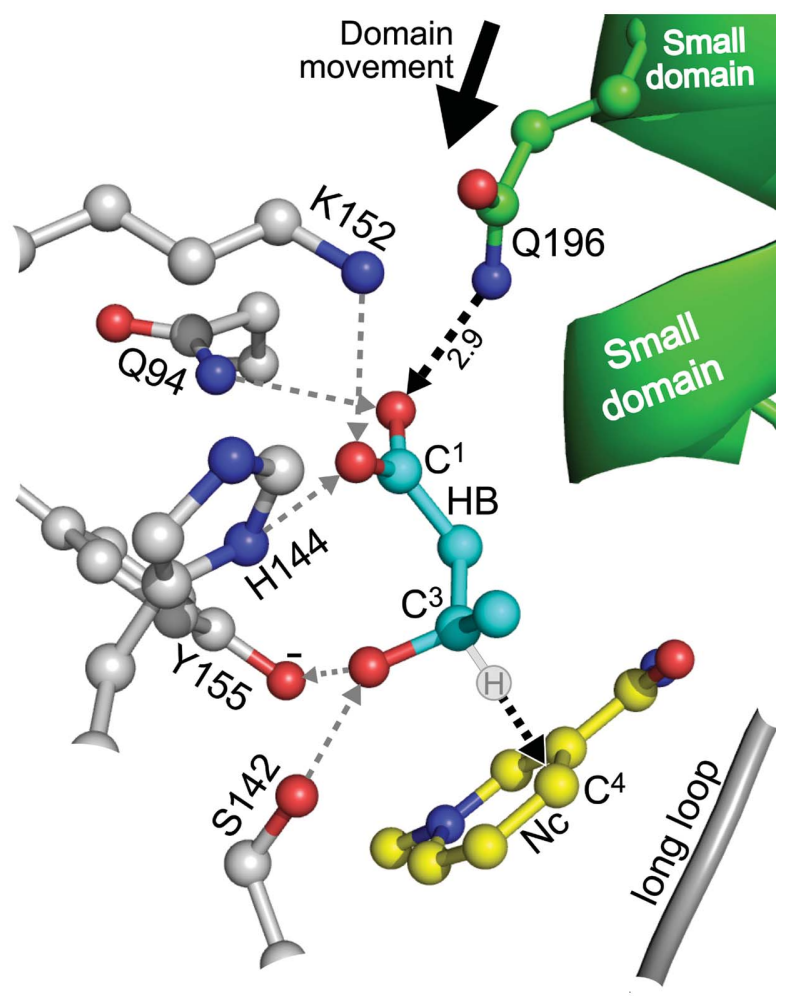

(c)

Figure 6

(a) Postulated donor-acceptor relationship of hydrogen bonds around Tyr155 of HBDH. The two arrows suggest H-atom movement in the catalytic reaction. (b) A comparison of 13 superposed HBDH monomers. Thin lines indicate Gln196 and thick lines indicate $\mathrm{C}^{\alpha}$ chains. When NAD ${ }^{+}$and $\mathrm{HB}$ are bound to HBDH, the small domain moves to close the active site (red) from the open form (black) by forming a hydrogen bond between Gln196 and HB. Stick lines and thin lines indicate $\mathrm{C}^{\alpha}$ chains and Gln196, respectively. As shown in $(c)$, which is viewed from the back side of $(b)$, the participation of the small domain in forming an additional hydrogen bond appears to facilitate the approach of the $\mathrm{H}$ atom of $\mathrm{C}^{3}$ to $\mathrm{C}^{4}$ of $\mathrm{Nc}$. 
bound through four hydrogen bonds in the same way as described above for the $\mathrm{HB}$ complex. A remarkable difference is found in one of the two $\mathrm{O}$ atoms, $\mathrm{O}^{3}$ and $\mathrm{O}^{4}$, of the second carboxylate group. The $\mathrm{O}^{4}$ atom occupies the hydrophobic pocket, similar to the methyl group of HB. In addition, it is accompanied by an isolated water molecule to form a hydrogen bond. In other words, MA mimics HB in shape with the help of an isolated water molecule to fill the void space. Therefore, the conformation of MA is similar to that of HB.

MA and mMA differ in the presence or the absence of a methyl group at the $\mathrm{C}^{2}$ atom. In the case of the mMA complex (Fig. $5 d$ ) the principal carboxylate group is similarly bound to
Gln94, Gln196 and Lys152 but not to His144. An H atom, postulated geometrically to be the $\mathrm{N}^{\delta}$ atom of His144, points in the direction of the $\mathrm{O}^{4}$ atom of mMA. The clearest difference from the MA complex is found in the methyl group, which is bound in the hydrophobic pocket. It implies that the hydrophobic effect is preferred over other hydrophilic interactions to comfortably fit mMA in the pocket. For this, as shown in Fig. 8(a), the conformation of the second carboxylate group is largely changed by a rotation of $79^{\circ}$ around the $\mathrm{C}^{1}-\mathrm{C}^{2}$ bond so that the methyl group approaches the position of the methyl group of $\mathrm{HB}$ and $\mathrm{O}^{4}$ is left near the $\mathrm{O}^{3}$ atom of MA. As a result, the $\mathrm{O}^{4}$ atom forms two hydrogen bonds to

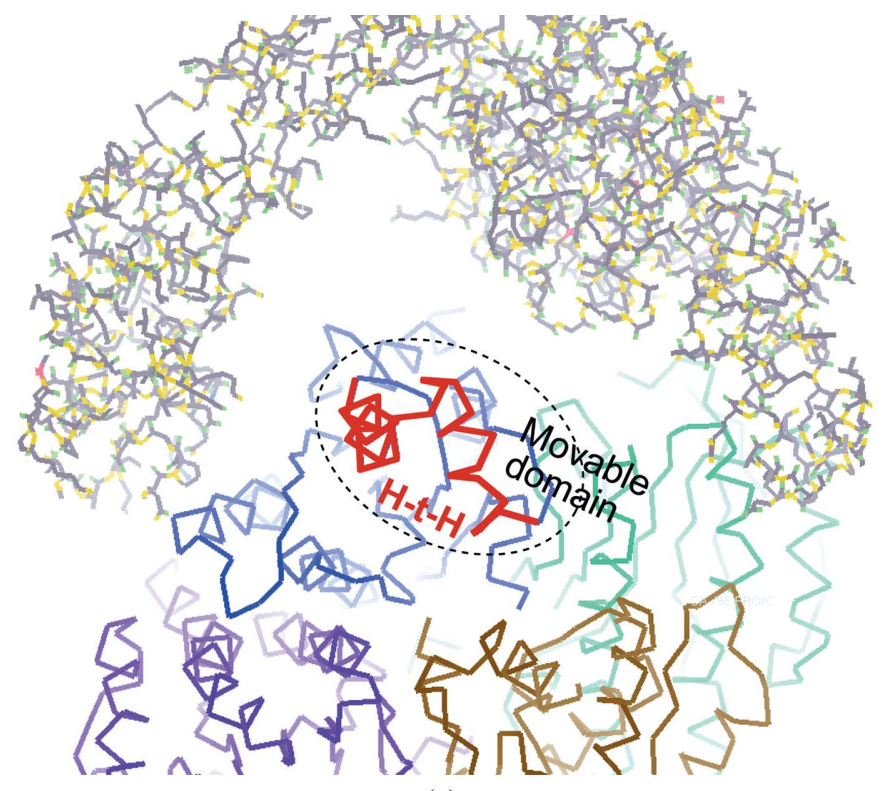

(a)

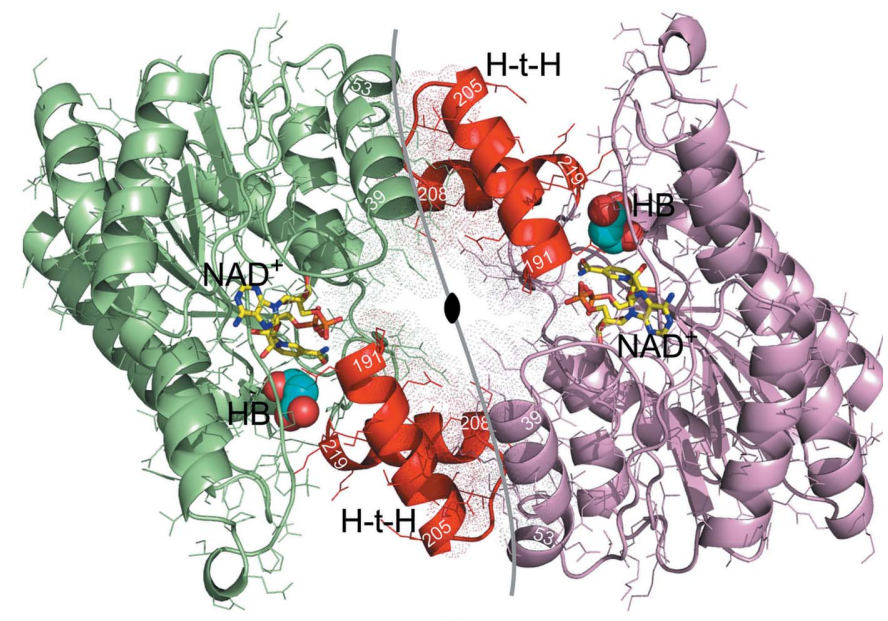

(b)

Figure 7

Comparison of the effects of the surroundings on the movable domain composed of a helix-turn-helix motif $(\mathrm{H}-\mathrm{t}-\mathrm{H})$ in the $P 4{ }_{1} 2{ }_{1} 2$ form $(a)$ and the $P 4_{2} 2_{1} 2$ form $(b)$. In $(a)$ the four subunits (violet, blue, green and brown) are drawn as $C^{\alpha}$ chains. The movable domain (red) is extruded into the solvent region, but it does not directly interact with the other tetramers. In $(b)$ (ribbon drawing) the two subunits of the neighbouring tetramers are associated at the interface (grey line), where the movable domain interacts with a rigid helix of another tetramer through direct hydrogen bonds and hydrophobic effects. Thin lines indicate side chains protruding from the ribbon models and dotted spheres indicate contacts between the movable domain and the main domain.

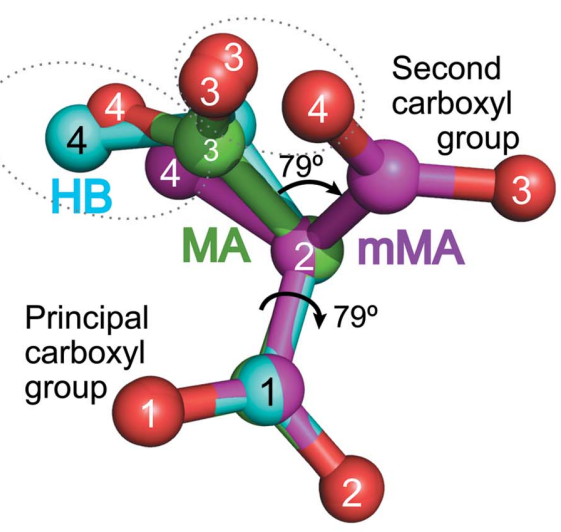

(a)

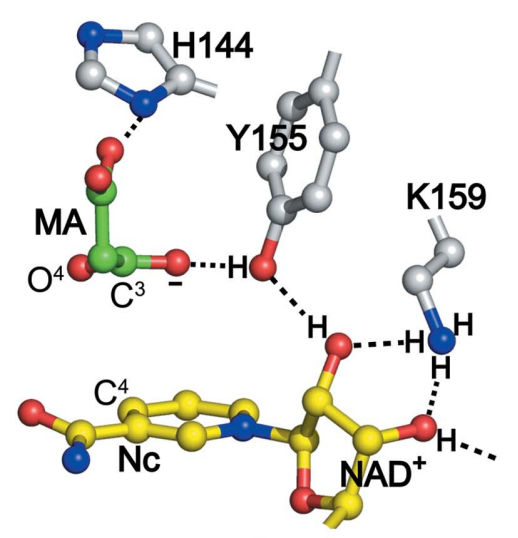

(b)

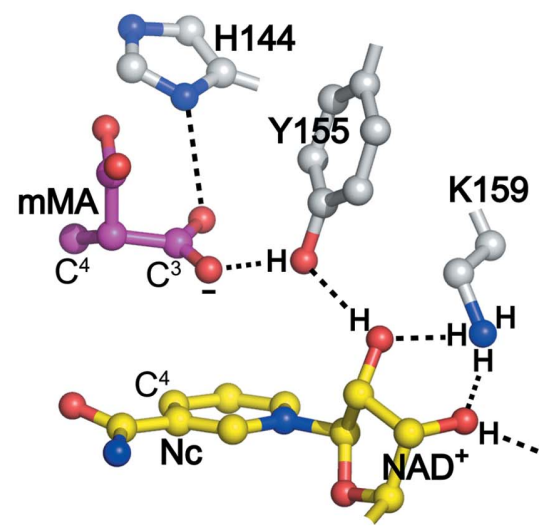

(c)

Figure 8

HB (cyan), MA (green) and mMA (purple) molecules bound in HBDH (a) and a possible H-atom arrangement for the hydrogen-bonded network of the MA complex $(b)$ and the mMA complex $(c)$. In $(a)$, the principal carboxylate groups of MA and mMA are superimposed on that of HB for comparison of conformational change. The second carboxylate group of mMA is rotated to accommodate the methyl group in the hydrophobic pocket. 
Ser142 and His144, and the $\mathrm{O}^{3}$ atom then forms a hydrogen bond to Tyr155.

Based on the above structural features, it is interesting to consider how MA and mMA inhibit the catalytic reaction. The main reason is simply that their carboxy groups positioned near the reaction centre have no active $\mathrm{H}$ atom to attack the $\mathrm{C}^{4}$ atom of Nc. Another factor involves the hydroxy group of Tyr155. Since the second carboxy groups of MA and mMA are also dissociated, they can behave as acceptors (see Figs. $8 b$ and $8 c$ ). To form another hydrogen bond, Tyr155 must accept the ribose hydroxy group. In both the MA complex and the mMA complex, therefore, the hydroxy group of Tyr155 should be undissociated to donate an $\mathrm{H}$ atom for hydrogen-bond formation to the carboxylate $\mathrm{O}$ atoms. In the case of L-HB, although it can bind to HBDH with a similar geometry and Tyr155 may be ionized in the hydrogen-bonded network as deduced from the deposited PDB data (PDB entry 2ztl; see Supplementary Fig. S3), the $\mathrm{H}$ atom of $\mathrm{C}^{3}$ is unfortunately not positioned in the direction of the $\mathrm{Nc} \mathrm{C}^{4}$ atom.

\section{Acknowledgements}

This work was supported in part by Grants-in-Aid for the Protein3000 Research Program from the Ministry of Education, Culture, Sports, Science and Technology of Japan. We thank S. Kuramitsu for organizing the research group in the program, and N. Igarashi and S. Wakatsuki for facilities and help during data collection.

\section{References}

Battye, T. G. G., Kontogiannis, L., Johnson, O., Powell, H. R. \& Leslie, A. G. W. (2011). Acta Cryst. D67, 271-281.

Brünger, A. T. (1992). Nature (London), 355, 472-475.

Brünger, A. T. (2007). Nature Protoc. 2, 2728-2733.

Brünger, A. T., Adams, P. D., Clore, G. M., DeLano, W. L., Gros, P., Grosse-Kunstleve, R. W., Jiang, J.-S., Kuszewski, J., Nilges, M., Pannu, N. S., Read, R. J., Rice, L. M., Simonson, T. \& Warren, G. L. (1998). Acta Cryst. D54, 905-921.

Dedkova, E. N. \& Blatter, L. A. (2014). Front. Physiol. 5, 260.

DeLano, W. L. (2002). PyMOL. http://www.pymol.org.

El Kebbaj, M. S., Latruffe, N. \& Gaudemer, Y. (1980). Biochem. Biophys. Res. Commun. 96, 1569-1578.
Emsley, P., Lohkamp, B., Scott, W. G. \& Cowtan, K. (2010). Acta Cryst. D66, 486-501.

Evans, P. (2006). Acta Cryst. D62, 72-82.

Guo, K., Lukacik, P., Papagrigoriou, E., Meier, M., Lee, W. H., Adamski, J. \& Oppermann, U. (2006). J. Biol. Chem. 281, 1029110297.

Harano, Y., Kosugi, K., Hyosu, T., Uno, S., Ichikawa, Y. \& Shigeta, Y. (1983). Clin. Chim. Acta, 134, 327-336.

Hoque, M. M., Shimizu, S., Hossain, M. T., Yamamoto, T., Imamura, S., Suzuki, K., Tsunoda, M., Amano, H., Sekiguchi, T. \& Takénaka, A. (2008). Acta Cryst. D64, 496-505.

Hoque, M. M., Shimizu, S., Juan, E. C. M., Sato, Y., Hossain, M. T., Yamamoto, T., Imamura, S., Suzuki, K., Amano, H., Sekiguchi, T., Tsunoda, M. \& Takénaka, A. (2009). Acta Cryst. F65, 331-335.

Ito, K., Nakajima, Y., Ichihara, E., Ogawa, K., Katayama, N., Nakashima, K. \& Yoshimoto, T. (2006). J. Mol. Biol. 355, 722-733.

Kavanagh, K. L., Jörnvall, H., Persson, B. \& Oppermann, U. (2008). Cell. Mol. Life Sci. 65, 3895-3906.

Koch, D. D. \& Feldbruegge, D. H. (1987). Clin. Chem. 33, 1761-1766.

Kwan, R. C. H., Hon, P. Y. T., Mak, W. C., Law, L. Y., Hu, J. \& Renneberg, R. (2006). Biosens. Bioelectron. 21, 1101-1106.

Laffel, L. (1999). Diabetes Metab. Res. Rev. 15, 412-426.

Mountassif, D., Andreoletti, P., Cherkaoui-Malki, M., Latruffe, N. \& El Kebbaj, M. S. (2010). Curr. Microbiol. 61, 7-12.

Murshudov, G. N., Skubák, P., Lebedev, A. A., Pannu, N. S., Steiner, R. A., Nicholls, R. A., Winn, M. D., Long, F. \& Vagin, A. A. (2011). Acta Cryst. D67, 355-367.

Murshudov, G. N., Vagin, A. A. \& Dodson, E. J. (1997). Acta Cryst. D53, 240-255.

Nakashima, K., Ito, K., Nakajima, Y., Yamazawa, R., Miyakawa, S. \& Yoshimoto, T. (2009). J. Biochem. 145, 467-479.

Navaza, J. (1994). Acta Cryst. A50, 157-163.

Paithankar, K. S., Feller, C., Kuettner, E. B., Keim, A., Grunow, M. \& Sträter, N. (2007). FEBS J. 274, 5767-5779.

Persson, B. \& Kallberg, Y. (2013). Chem. Biol. Interact. 202, 111-115. Philippsen, A. (2003). DINO. http://www.dino3d.org.

Pires, C. K., Martelli, P. B., Reis, B. F., Lima, J. L. F. C. \& Saraiva, M. L. M. F. S. (2003). J. Agric. Food Chem. 51, 2457-2460.

Shimomura, T., Sumiya, T., Ono, M., Ito, T. \& Hanaoka, T. (2013). Anal. Bioanal. Chem. 405, 297-305.

Shuster, C. W. \& Doudoroff, M. (1962). J. Biol. Chem. 237, 603-607.

Tan, A. W. H., Smith, C. M., Aogaichi, T. \& Plaut, G. W. E. (1975). Arch. Biochem. Biophys. 166, 164-173.

Uno, S., Ito, S., Kurono, M., Yamaoka, Y., Kamiyama, Y. \& Ozawa, K. (1987). Clin. Chim. Acta, 168, 253-255.

Vagin, A. \& Teplyakov, A. (2010). Acta Cryst. D66, 22-25.

Williamson, D. H., Mellanby, J. \& Krebs, H. A. (1962). Biochem. J. 82, 90-96.

Winn, M. D. et al. (2011). Acta Cryst. D67, 235-242. 